Ophthalmologe 2022 119 (Suppl 1):S1-S2 https://doi.org/10.1007/s00347-021-01564-5 Angenommen: 17. Dezember 2021

(c) The Author(s), under exclusive licence to Springer Medizin Verlag GmbH, ein Teil von Springer Nature 2021

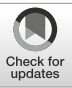

\title{
Mit Wissenschaft ins neue Jahr. Frei eingereichte Artikel in Der Ophthalmologe
}

Frank G. Holz ${ }^{1}$ Michal Meyer zu Tittingdorf ${ }^{2}$

'Universitäts-Augenklinik Bonn, Bonn, Deutschland

${ }^{2}$ Springer Medizin Verlag GmbH, Heidelberg, Deutschland

Liebe Leserinnen, liebe Leser,

Sie halten mit dieser Ausgabe eine Besonderheit in den Händen: ein ganzes Sonderheft voll von hervorragenden Originalarbeiten, die einen Einblick in die hochkarätige deutsche, ophthalmologische Forschungslandschaft geben, sowie spannenden Fallbeispielen aus dem Klinik- und Praxisalltag.

Diese Ausgabe erscheint 2022 zusätzlich zu den regulären 12 Ausgaben von Der Ophthalmologe, um den zunehmenden Einreichungen bei unserer Zeitschrift Rechnung zu tragen. So freuen wir uns seit Jahren über stetig mehr qualitativ gute Einreichungen, die wir gerne in unserer Zeitschrift neben der Online-Verfügbarkeit abdrucken möchten, aufgrund des begrenzten Platzes jedoch immer seltener zeitnah abdrucken können.

Auch die Januar-Ausgabe jeden Jahres widmen wir als sog. „Originalien-Ausgabe" den frei eingereichten Übersichten, Originalien, Kasuistiken und Bild und FallBeiträgen, sodass Sie in diesem Januar nahezu gleichzeitig 2 umfangreiche Ausgaben erhalten, die eindrucksvoll die sehr gut aufgestellten Forschungsaktivitäten in Deutschland widerspiegeln.

Zusätzlich zu dieser Sonderausgabe werden wir in diesem Jahr auch den Umfang der regulären Ausgaben erhöhen, um Ihnen monatlich mehr Beiträge pro Ausgabe bieten zu können.

In einer Analyse über die letzten Jahre hat sich gezeigt, dass auch die Länge der Beiträge in den letzten Jahren kontinuierlich gestiegen ist - ein weiterer Punkt, warum wir nicht alle frei eingereichten Beiträge entsprechend zeitnah abdrucken können. Wir werden daher - auch ganz im Sinne unserer Leserinnen und Leser zukünftig verstärkt auf die Einhaltung der formalen Kriterien wie Beitragslänge, Anzahl der Abbildungen und Literaturzitate achten. Am Ende dieses Editorials finden Sie eine entsprechende Übersicht über die Vorgaben für die einzelnen Rubriken. Wir freuen uns, wenn Sie dies bei Ihrer nächsten Einreichung beachten!

\section{》) Für Leitthemen und "CME Zertifizierte Fortbildung" sind Themenwünsche stets willkommen}

Liebe Leserinnen, liebe Leser, wenn Sie sich für unsere Leitthemen und die Rubrik "CME Zertifizierte Fortbildung" Themen wünschen, senden Sie diese gerne an die Verlagsredaktion, und wir prüfen, ob und wie wir dies möglich machen können. Auch freuen wir uns, wenn Sie uns für unsere Rubrik "Ophthalmologischer Schnappschuss" Ihre spannendsten Aufnahmen oder klinisch-pathologische Korrelationen mit einer prägnanten und aussagekräftigen Bildlegende zusenden! Und denken Sie an Ihre Operationsvideos. Reichen Sie diese einfach zusätzlich zu Ihrem Beitrag bei uns ein oder machen Sie es bei einer bewährten operativen Technik ganz zum Kern des Beitrags und senden Sie es uns für die Rubrik "Video plus".

Wir wünschen Ihnen beim Lesen dieses Sonderheftes viele Denkanstöße und Anregungen. Wir bedanken uns ganz besonders bei allen Autorinnen und Autoren für ihre wertvollen Beiträge.

Ihre

Frank G. Holz

Michal Meyer zu Tittingdorf 


\section{In eigener Sache}

\section{Korrespondenzadresse}

Michal Meyer zu Tittingdorf

Springer Medizin Verlag GmbH

Tiergartenstr. 17, 69121 Heidelberg,

Deutschland

michal.meyerzutittingdorf@springer.com

Interessenkonflikt. F.G. Holz und M. Meyer zu Tittingdorf geben an, dass kein Interessenkonflikt besteht.

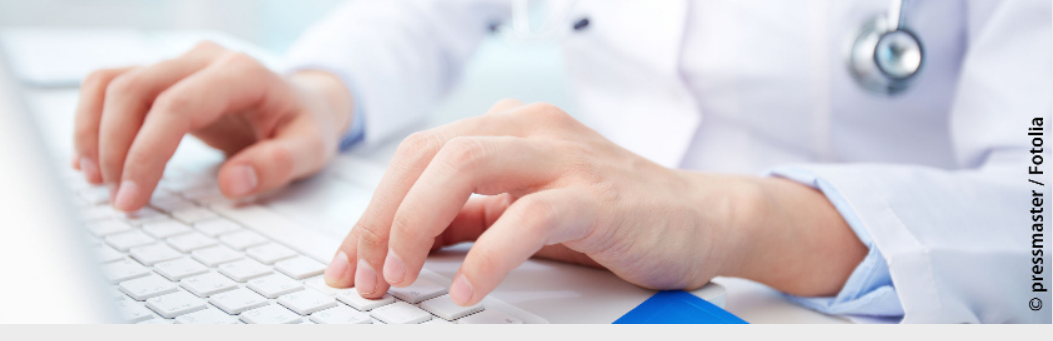

Für Autor*innen: Ihr Beitrag in Der Ophthalmologe

Das sollten Sie als Autor*in beachten

Wir freuen uns über jede Einreichung in den Rubriken „Originalien“, „Übersichten“, "Kasuistiken" und „Bild und Fall".

Bitte beachten Sie bei der Erstellung Ihres Beitrags folgende Formatvorgaben:

\section{Originalien}

- Vorstellung und Diskussion von Methoden und Ergebnissen eigener Studien

- Gliederung: Hintergrund, Methoden, Ergebnisse, Diskussion

- max. 25.000 Zeichen inkl. Leerzeichen (Literatur, Tabellen und Abbildungslegenden bitte mitzählen)

- Kurze gegliederte Zusammenfassung (deutsch und englisch)

- 5 Schlüsselwörter (deutsch und englisch)

- Max. 6 Abbildungen inkl. Verweise im Text

- Max. 30 Literaturstellen

\section{Übersichten}

- Kritische Zusammenstellung und Diskussion relevanter wissenschaftlicher Ergebnisse

- max. 25.000 Zeichen inkl. Leerzeichen (Literatur, Tabellen und Abbildungslegenden bitte mitzählen)

- Kurze Zusammenfassung (deutsch und englisch)

- 5 Schlüsselwörter (deutsch und englisch)

- Max. 6 Abbildungen inkl. Verweise im Text

- Max. 30 Literaturstellen

\section{Kasuistiken}

- Kurzes Fallbeispiel, das Besonderheiten der klinischen Praxis, Fallstricke der Diagnostik und ungewöhnliche Krankheits- und Behandlungsverläufe aufzeigt

- Gliederung: Anamnese, Befund, Diagnose, Therapie und Verlauf, Diskussion

- max. 10.000 Zeichen inkl. Leerzeichen (Literatur, Tabellen und Abbildungslegenden bitte mitzählen)

- ohne Zusammenfassung und Schlüsselwörter

- Max. 6 Abbildungen inkl. Verweise im Text

- Max. 10 Literaturstellen

\section{Bild und Fall}

- Kurzes Fallquiz, bei dem der Leser miträtseln kann

- Gliederung: Teil 1 - Falldarstellung mit Anamnese, klinischem Befund und Diagnostik. Teil 2 - Diagnose, Therapie und Verlauf, ggf. weitere Hintergrundinformationen

- max. 10.000 Zeichen inkl. Leerzeichen (Literatur, Tabellen und Abbildungslegenden bitte mitzählen)

- ohne Zusammenfassung und Schlüsselwörter

- Max. 5 Abbildungen inkl. Verweise im Text

- Max. 5 Literaturstellen

Ausführliche Leitfäden finden Sie unter www.DerOphthalmologe.de 